\title{
Primary non-Hodgkin's lymphoma of the vagina: A case report
}

\author{
FENG WANG ${ }^{1,2}$, XUQUAN JING ${ }^{2}$, BO LIU ${ }^{3}$, XUE MENG ${ }^{2}$, XINDONG SUN ${ }^{2}$, \\ YONGSHENG GAO ${ }^{4}$, LINLIN WANG ${ }^{2}$ and ZHENG FU ${ }^{5}$

\begin{abstract}
${ }^{1}$ School of Medicine and Life Sciences, University of Jinan-Shandong Academy of Medical Sciences, Jinan, Shandong 250200; Departments of ${ }^{2}$ Radiation Oncology, ${ }^{3}$ Medical Oncology, ${ }^{4}$ Pathology and ${ }^{5}$ Computed Tomography, Shandong Cancer Hospital Affiliated to Shandong University, Jinan, Shandong 250117, P.R. China
\end{abstract}

Received November 22, 2016; Accepted November 7, 2017

DOI: $10.3892 / \mathrm{ol} .2018 .7805$

\begin{abstract}
Primary non-Hodgkin's lymphoma (NHL) of the vagina is uncommon. The present case study reports the case of a 54-year-old female with a palpable mass between the rectum and vagina. The patient presented with symptoms consistent with vaginal cancer but lacked any of the 'B' symptoms often associated with systemic lymphoma, including fever, weight loss, night sweats and fatigue. The mass was resected under anesthesia. Immunohistochemistry and biopsy confirmed diffuse large B-cell NHL (DLBCL). Following surgery, six cycles of chemotherapy with rituximab, cyclophosphamide, doxorubicin, vincristine and prednisone were administered. Subsequently, a vertebral body metastasis was observed using a computed tomography scan and whole-body bone imaging. The patient received palliative radiation for the vertebral body metastasis. Additionally, the available literature was reviewed in order to further characterize this rare disease.
\end{abstract}

\section{Introduction}

Primary vaginal cancer, including squamous cell carcinoma, adenocarcinoma, melanoma and sarcoma, is an uncommon type of malignancy. By convention, if both the cervix and vagina are involved, the primary diagnosis is cervical cancer. Surgery serves an important role in the treatment of primary vaginal and cervical cancer in patients with early stage disease $(1,2)$. The 5 -year survival rate for stage I carcinomas is $73 \%$ after surgical therapy (1).

Correspondence to: Professor Bo Liu, Department of Medical Oncology, Shandong Cancer Hospital Affiliated to Shandong University, 440 Jiyan Road, Jinan, Shandong 250117, P.R. China E-mail: 15553115688@163.com

Dr Xue Meng, Department of Radiation Oncology, Shandong Cancer Hospital Affiliated to Shandong University, 440 Jiyan Road, Jinan, Shandong 250117, P.R. China

E-mail: 755700894@qq.com

Key words: vagina cancer, primary extra-nodal non-Hodgkin's lymphoma, vaginal lymphoma
Non-Hodgkin's lymphoma (NHL) with the female genital tract as the primary site is rare; the National Cancer Database reported that only $1.5 \%$ of extra-nodal NHL originates in the female genital tract (3). Surgery does not serve a role in the treatment of this disease, as chemotherapy is relatively effective (4). As a result, it is important for gynecologists to be aware of this disease in the differential diagnosis of gynecological cancer. The present case report illustrates the presentation, diagnosis and appropriate treatment of primary extra-nodal non-Hodgkin's lymphoma of the vagina (PNHLV).

\section{Case report}

A 54-year-old woman was referred to the gynecology outpatient clinic of Shandong Cancer Hospital Affiliated to Shandong University with a 2-month history of vaginal bleeding. The patient provided written informed consent to participate in the present case report. The patient did not report any other symptoms, including fever, night sweats, weight loss or fatigue ('B' symptoms), and they had no notable past medical history. Upon digital rectal examination, a palpable mass was discovered between the rectum and vagina, apparently invading the rectal mucosa. The colposcopy revealed a thickening of the posterior vaginal wall. Magnetic resonance imaging (MRI) (Fig. 1) of the pelvis and ultrasonography revealed a diffuse mass with a diameter of $\sim 6 \mathrm{~cm}$ in the external cervical orifice and invading the vagina. The uterus and bladder were not involved.

On admission, the following laboratory parameters were noted: Blood cell count 4.1 x10 $12 / 1$ (normal range, $4.0-5.0 \times 10^{12} / 1$ ); dehydrogenase $181 \mathrm{U} / 1$ (normal range, 109-245 U/1); and cancer antigen-125; $29 \mathrm{kU} / \mathrm{ml}$ (normal range, 0-35.0 kU/ml). Baseline renal and liver function test results were also normal. Hepatitis B virus markers, hepatitis $\mathrm{C}$ virus antibody $(\mathrm{Ab})$ and human immunodeficiency virus Ab tests were negative. Based on physical examination and laboratory findings, a surgical exploration was performed following a suspected diagnosis of cervical carcinoma or primary vaginal carcinoma. The mass was resected from the patient under anesthesia (Fig. 2). Immunohistochemistry and biopsy confirmed the lymphoid origin of the neoplasm (Fig. 3), and tumor cells were positive for cluster of differentiation 20 (CD20) (Fig. 4) and CD79a (Fig. 5). The bone marrow aspirate and biopsy did not reveal 


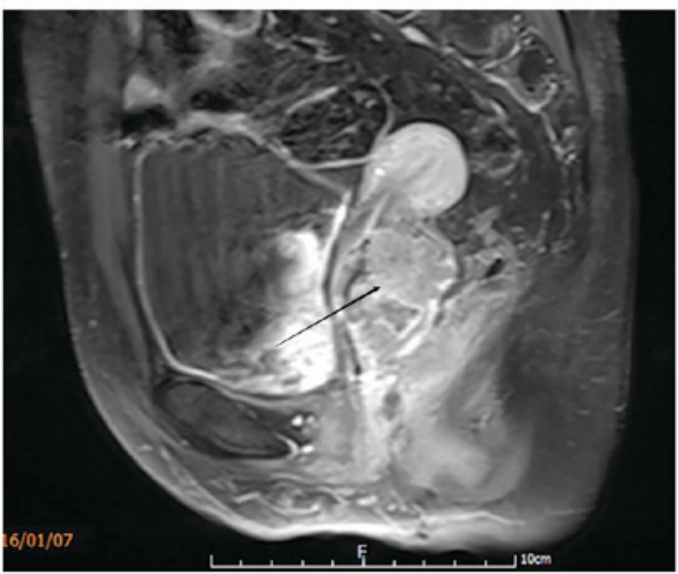

Figure 1. MRI of the pelvis (sagittal view). MRI, magnetic resonance imaging.

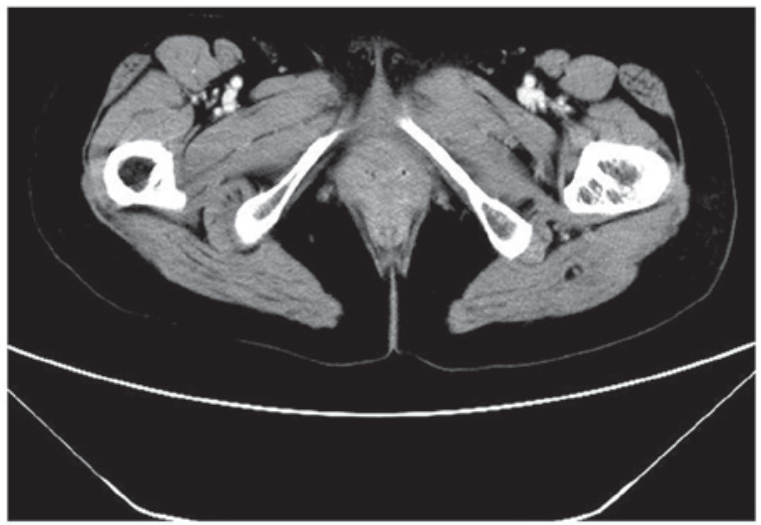

Figure 2. Postoperative CT of the pelvis (coronal view). CT, computed tomography.

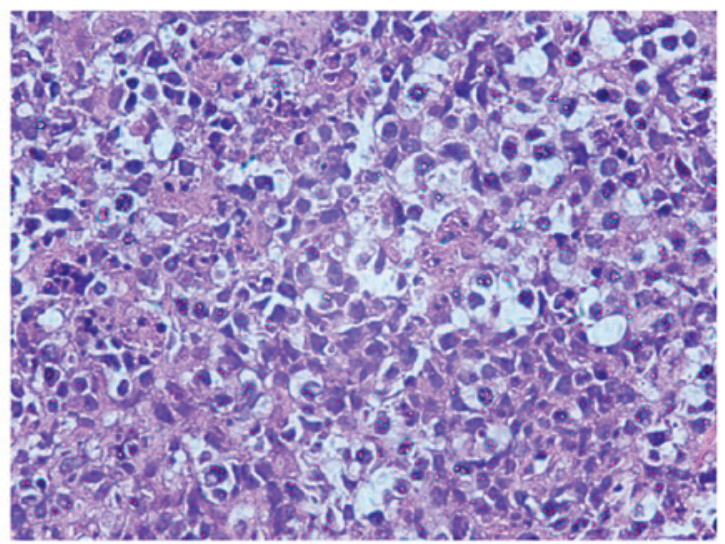

Figure 3. Vaginal biopsy. The infiltrate is composed of a mixture of small cleaved and large lymphocytes. Magnification, x200.

lymphomatous infiltration. A computed tomography (CT) scan of the chest and abdomen did not detect metastasis to other locations. The patient was diagnosed with stage IE primary vaginal large B-cell NHL disease based on the Ann Arbor system (5) for staging NHL. One month after the surgery, oncologists reviewed the patient and recommended 6 cycles of the CHOP regime (1,000 mg cyclophosphamide on days 1 and

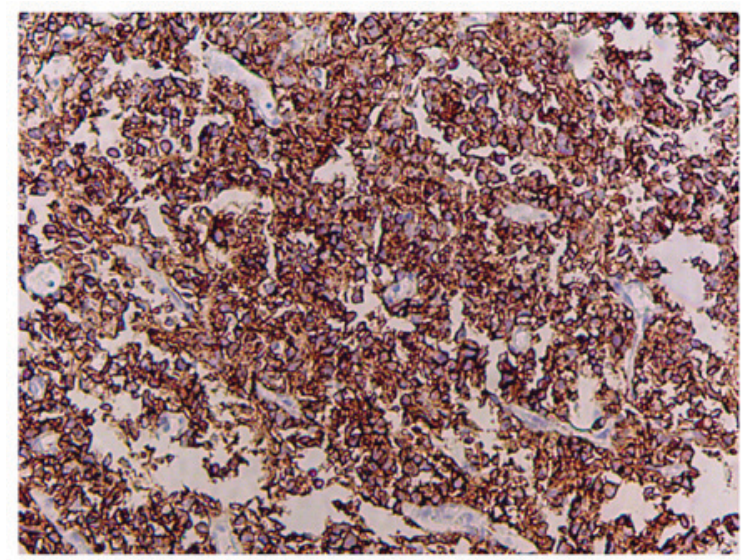

Figure 4. Malignant B cells staining positive for CD20. Magnification, x200. CD20, cluster of differentiation 20 .

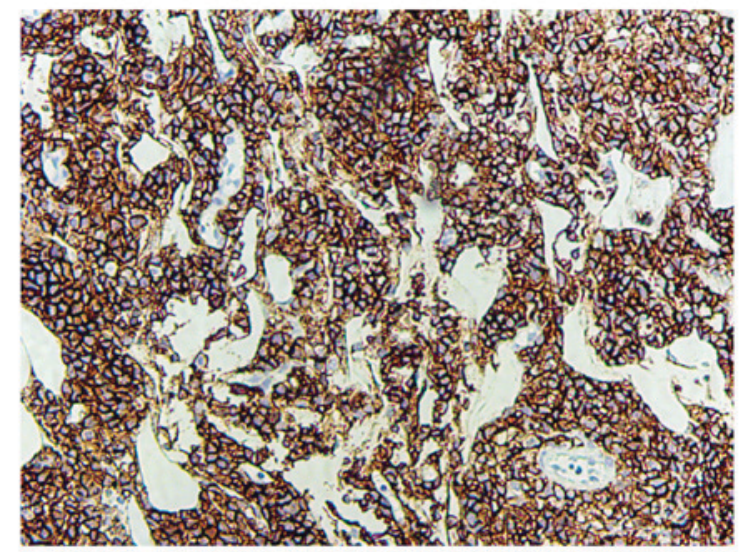

Figure 5. Malignant B cells staining positive for CD79a. Magnification, x200. CD79a, cluster of differentiation 79a.

$8,50 \mathrm{mg}$ doxorubicin on day 1 , vincristine $2 \mathrm{mg}$ on day 1 and $100 \mathrm{mg}$ prednisone on days 1-5 for 21 days/cycle). Rituximab was not used in the chemotherapy regimen for financial reasons.

Following 2 cycles of chemotherapy, CT scans of the pelvis, abdomen and thorax were negative. Following the 6 cycles of chemotherapy, a CT scan (Fig. 6) and whole-body bone imaging (Fig. 7) revealed evidence of thoracic vertebral body metastasis. However, the abdomen and pelvis were negative for NHL. The patient received palliative radiation (36 Gy in 20 fractions) for the vertebral body metastasis. At time of publication, the patient was reviewed every three months and no recurrence or other metastasis had been observed.

\section{Discussion}

Fewer than $0.5 \%$ of all cases of extra-nodal NHL involve the female genital tract (5). Chorlton et al (6) reviewed 9,500 cases of lymphomas in women and observed only 4 cases of primary vaginal lymphoma. Primary vaginal lymphomas are reported in patients from a wide range of ages, with a mean age at presentation of 42 years (range, 26-66 years) (7).

Guastafierro et al (8) identified that 63 patients with primary extra-nodal NHL of the vagina were reported between 1954 


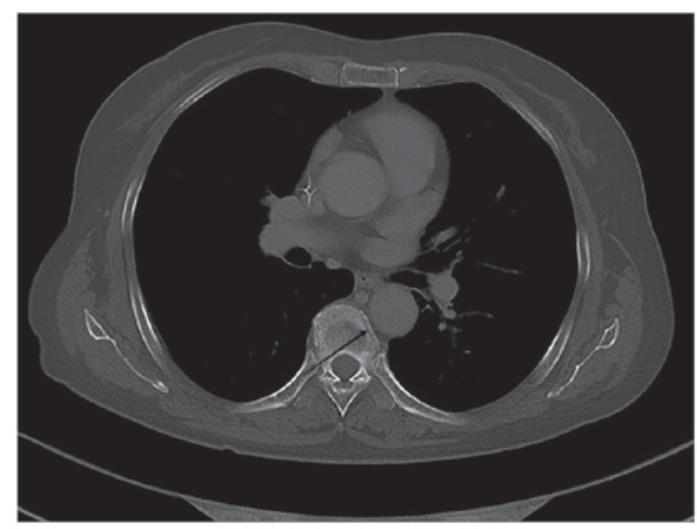

Figure 6. CT of a thoracic vertebral body metastasis, which exhibited a low signal on T1-weighted imaging. CT, computed tomography.

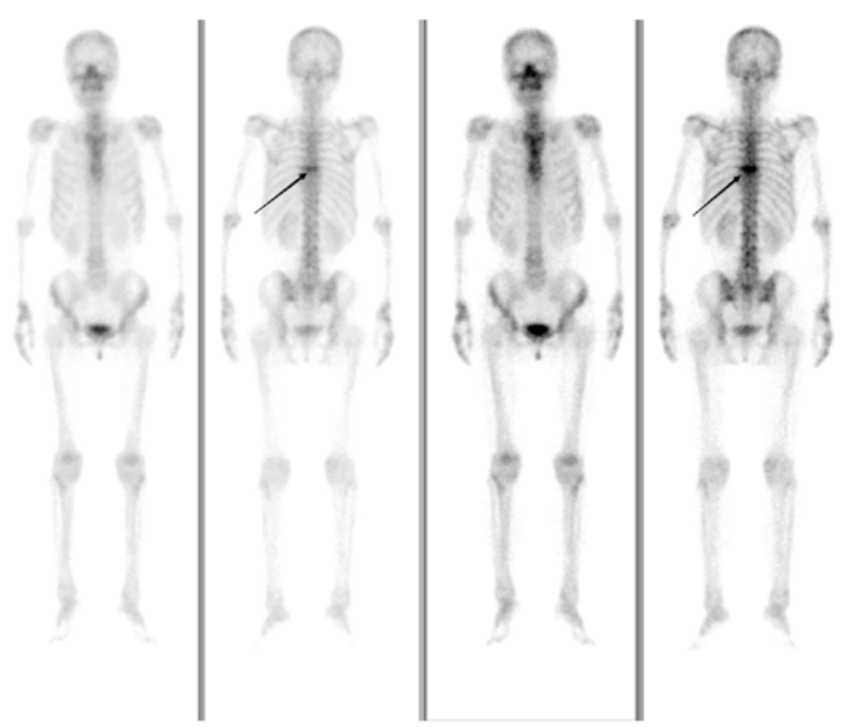

Figure 7. Whole body bone imaging following chemotherapy.

and 2012. The most common histological feature was diffuse large B-cell NHL (DLBCL; 40/58 evaluable cases, 69\%). At diagnosis, the most common clinical presentations were vaginal bleeding (34.8\%), vaginal masses (23.9\%), vaginal discharge (17.4\%) and abdominal and/or pelvic pain (15.2\%), with $13 \%$ being fully asymptomatic (8). As indicated in the present case report, 'B' symptoms at diagnosis of NHL of the vagina are exceedingly rare compared with other lymphomas. Therefore, non-specific clinical findings usually delay the diagnosis of this disease.

In general, the prognosis for extra-nodal lymphoma is worse than nodal lymphoma, primarily due to the inaccurate or delayed diagnosis and inadequate treatment (3). It is important for gynecologists to be aware of this disease in the differential diagnosis of gynecological cancer. In practice, irregular vaginal bleeding is often indicative of gynecological pathology, with the initial diagnostic step a Pap smear test and/or colposcopy. In DLBCL, sheets of neoplastic cells infiltrate deeply into the stroma, producing a dense sclerosis. The lymphoma cells lack tropism for the overlying epithelium, and lymphoepithelial lesions are not observed (9). The sensitivity of a conventional Pap smear for the detection of malignant lymphoma is low, ranging between 20 and $30 \%$ (10). Colposcopy results are unremarkable (11), and may mislead clinicians, as in the case of the subject of the present case report.

Zeppa et al (12) demonstrated that fine-needle aspiration cytology (FNAC) coupled with flow cytometry is effective in the cytological diagnosis and classification of NHL with reproducible results. However, the lack of diagnostic material and other clinicopathological limitations may represent an obstacle to achieving accurate diagnosis. As a result, a biopsy is the preferable option, while FNAC may be performed in patients for whom excisional biopsy is contraindicated, or in the case of ancillary studies (8). In the present case report, NHL was not suspected clinically and may have been missed without biopsy and tissue diagnosis.

The differential diagnosis of vaginal NHL includes other hematopoietic lesions (including granulocytic sarcoma and Langerhan's cell histiocytosis), carcinoma, malignant mixed Mullerian tumor, epithelioid leiomyosarcoma and endometrial stromal tumors, including endometrial stromal sarcoma, melanoma and extraosseous Ewing's sarcoma/primitive neuroectodermal tumor (13). The pathological diagnosis of DLBCL is based on morphology and immunophenotyping. DLBCL is a heterogeneous group of tumors consisting of large, transformed B cells with prominent nucleoli, a basophilic cytoplasm, a diffuse growth pattern and a high proliferation fraction. Tumor cells generally express pan-B cell antigens, including CD19, CD20, CD22 and CD79a (14). A screening panel consisting of CD45, CD3, CD20 and cytokeratin is useful in determining whether the tumor is a B-cell lymphoma (CD20+), T-cell lymphoma (CD3+), granulocytic sarcoma (only CD45+) or carcinoma (cytokeratin+) (9).

$\mathrm{CT}$ and/or positron emission tomography (PET) imaging of the pelvis may provide valuable information regarding the sites involved in NHL, particularly with respect to nodular involvement. In addition, these techniques, in conjunction with bone marrow biopsy, aid in the staging of disease $(4,15)$. Initial imaging serves both to determine disease stage at diagnosis and to provide baseline measurements for comparison to determine the response to treatment (14).

It is important to recognize the prognostic factors of NHL in order to identify the patients who may be refractory to standard therapy as early as possible and to enable them to benefit from more intensive treatments. PET scanning has been used for the surveillance and assessment of the response to treatment (16). The end-of-treatment PET scan is highly predictive of progression-free survival (PFS). Among 88 DLBCL patients treated with 6-8 courses of R-CHOP, patients with a negative final PET scan achieved a 2-year PFS of $83 \%$, compared with $64 \%$ for patients with a positive final PET scan $(\mathrm{P}<0.001)(17)$. Elevated serum levels of cancer antigen-125 at the time of diagnosis have been associated with a decreased 5-year survival rate (18). Vang et al (7) demonstrated that patients with low-stage vaginal NHL usually have an excellent prognosis.

On the other hand, the observation that the prognosis of extra-nodal NHL is worse than that for nodal lymphoma may be associated with substandard treatment (19). According to the National Cancer Database, only $46 \%$ of patients with extra-nodal lymphoma were treated with any chemotherapy, compared with $70 \%$ of patients with nodal lymphoma (3). 
There is no established treatment for primary NHL of the vagina, most likely due to its rarity. According to recent international guidelines (20), the treatment for early stage DLBCL is chemotherapy with R-CHOP with or without subsequent local radiation therapy. In a review by Signorelli et al (21), which evaluated 10 cases of extra-nodal genital lymphoma treated with the CHOP or R-CHOP regimen, 9 complete responses were observed. In a case reported by Guastafierro et al (8), the patient remained in complete remission for 72 months following chemotherapy with 8 cycles of the R-CHOP regime. Signorelli et al (21) reported on a patient with stage IEA NHL of the vagina who underwent complete remission following treatment with the MACOP-B regimen (methotrexate with leucovorin rescue, doxorubicin, cyclophosphamide, vincristine, prednisone and bleomycin), and the patient gave birth four years after the treatment. As these tumors are highly responsive to chemotherapy, surgery is not the primary mode of therapy for NHL (15).

In modern clinical practice, the $\mathrm{R}-\mathrm{CHOP}$ treatment regimen has proven to be highly effective and radiation therapy (RT) is slowly being phased out as a treatment option for DLBCL. Modern advanced imaging and conformal RT techniques now enable the treatment of larger and anatomically more challenging target volumes with much less radiation exposure than normal tissues and, consequently, much lower risks of long-term complications (22). Different forms of RT, including involved-field RT, involved-node RT and involved-site RT, may be used alongside these 'new-age' treatment strategies to further improve prognostic outcomes and overall survival rates. Nevertheless, RT remains an important treatment method and may be used instead of certain treatments, including the treatment of patients who are unresponsive to or develop complications from the use of chemotherapy (23). A study by Held et al (24) provided strong support for adding RT in the treatment of large $(\geq 7.5 \mathrm{~cm}$ in diameter) tumors in elderly patients ( $>60$ years of age) with aggressive B-cell lymphoma. However, it is unclear whether or not a positive post-chemotherapy PET scan can identify patients who are likely to benefit from RT (22). Therefore, RT may be reserved for chemo-resistant disease, disease failing to achieve remission following chemotherapy, or sites of large tumors.

In conclusion, primary NHL of the vagina occurs rarely. Early diagnosis and standard treatment lead to improved survival rates for the affected patients. The present case report aims to raise awareness of this rare disease, which must be considered in the differential diagnosis of genital tract diseases. Radical surgery does not serve a role in the treatment of vaginal NHL, as chemotherapy is relatively effective.

\section{References}

1. Creasman WT, Phillips JL and Menck HR: The national cancer data base report on cancer of the vagina. Cancer 83: 1033-1040, 1998.

2. Otton GR, Nicklin JL, Dickie GJ, Niedetzky P, Tripcony L, Perrin LC and Crandon AJ: Early-stage vaginal carcinoma-an analysis of 70 patients. Int J Gynecol Cancer 14: 304-310, 2004.

3. Trenhaile TR and Killackey MA: Primary pelvic non-Hodgkin's lymphoma. Obstet Gynecol 97: 717-720, 2001.

4. Ragupathy $\mathrm{K}$ and Bappa L: Primary vaginal non-Hodgkin lymphoma: Gynecologic diagnosis of a hematologic malignancy. J Low Genit Tract Dis 17: 326-329, 2013.
5. Lister TA, Crowther D, Sutcliffe SB, Glatstein E, Canellos GP, Young RC, Rosenberg SA, Coltman CA and Tubiana M: Report of a committee convened to discuss the evaluation and staging of patients with Hodgkin's disease: Cotswolds meeting. J Clin Oncol 7: 1630-1636, 1989.

6. Chorlton I, Karnei RF Jr, King FM and Norris HJ: Primary malignant reticuloendothelial disease involving the vagina, cervix, and corpus uteri. Obstet Gynecol 44: 735-748, 1974.

7. Vang R, Medeiros LJ, Silva EG, Gershenson DM and Deavers M: Non-Hodgkin's lymphoma involving the vagina: A clinicopathologic analysis of 14 patients. Am J Surg Pathol 24: 719-725, 2000.

8. Guastafierro S, Tedeschi A, Criscuolo C, Celentano M, Cobellis L, Rossiello R and Falcone U: Primary extranodal non-Hodgkin's lymphoma of the vagina: A case report and a review of the literature. Acta Haematol 128: 33-38, 2012.

9. Lagoo AS and Robboy SJ: Lymphoma of the female genital tract: Current status. Int J Gynecol Pathol 25: 1-21, 2006.

10. Harris NL and Scully RE: Malignant lymphoma and granulocytic sarcoma of the uterus and vagina. A clinicopathologic analysis of 27 cases. Cancer 53: 2530-2545, 1984.

11. Hanley KZ, Tadros TS, Briones AJ, Birdsong GG and Mosunjac MB: Hematologic malignancies of the female genital tract diagnosed on liquid-based Pap test: Cytomorphologic features and review of differential diagnoses. Diagn Cytopathol 37: 61-67, 2009.

12. Zeppa P, Vigliar E, Cozzolino I, Troncone G, Picardi M, De Renzo A, Grimaldi F, Pane F, Vetrani A and Palombini L: Fine needle aspiration cytology and flow ytometry immunophenotyping of non-Hodgkin lymphoma: Can we do better. Cytopathology 21: 300-310, 2010.

13. Vang R, Medeiros LJ, Ha CS and Deavers M: Non-Hodgkin's lymphomas involving the uterus: A clinicopathologic analysis of 26 cases. Mod Pathol 13: 19-28, 2000.

14. Silva V, Correia P, Oliveira N and Sá L: Primary vaginal non-Hodgkin's lymphoma: Report of a rare clinical entity. Clin Pract 5: 821, 2015.

15. Kendrick JE IV and Straughn JM Jr: Two cases of non-Hodgkin's lymphoma presenting as primary gynecologic malignancies. Gynecol Oncol 98: 490-492, 2005.

16. Lavely WC, Delbeke D, Greer JP, Morgan DS, Byrne DW, Price RR and Hallahan DE: FDG PET in the follow-up management of patients with newly diagnosed Hodgkin and non-Hodgkin lymphoma after first-line chemotherapy. Int J Radiat Oncol Biol Phys 57: 307-315, 2003.

17. Pregno P, Chiappella A, Bellò M, Botto B, Ferrero S, Franceschetti S, Giunta F, Ladetto M, Limerutti G, Menga M, et al: Interim 18-FDG-PET/CT failed to predict the outcome in diffuse large B-cell lymphoma patients treated at the diagnosis with rituximab-CHOP. Blood 119: 2066-2073, 2012.

18. Zacharos ID, Efstathiou SP, Petreli E, Georgiou G, Tsioulos DI, Mastorantonakis SE, Christakopoulou I and Roussou PP: The prognostic significance of CA 125 in patients with non-Hodgkin's lymphoma. Eur J Haematol 69: 221-226, 2002.

19. Cohn DE, Resnick KE, Eaton LA, deHart J and Zanagnolo V: Non-Hodgkin's lymphoma mimicking gynecological malignancies of the vagina and cervix: A report of four cases. Int $\mathbf{J}$ Gynecol Cancer 17: 274-279, 2007.

20. Tilly H, Gomes da Silva M, Vitolo U, Jack A, Meignan M, Lopez-Guillermo A, Walewski J, André M, Johnson PW, Pfreundschuh M, et al: Diffuse large B-cell lymphoma (DLBCL): ESMO Clinical Practice Guidelines for diagnosis, treatment and follow-up. Ann Oncol 26 (Suppl 5): v116-v125, 2015.

21. Signorelli M, Maneo A, Cammarota S, Isimbaldi G, Garcia Parra R, Perego P, Maria Pogliani E and Mangioni C: Conservative management in primary genital lymphomas: The role of chemotherapy. Gynecol Oncol 104: 416-421, 2007.

22. Specht L: Does radiation have a role in advanced stage Hodgkin's or non-Hodgkin lymphoma? Curr Treat Options Oncol 17: 4, 2016.

23. Mendes F, Domingues C, Teixo R, Abrantes AM, Gonçalves AC, Nobre-Gois I, Jacobetty M, Sarmento AB, Botelho MF and Rosa MS: The importance of radiotherapy on diffuse large B cell lymphoma treatment: A current review. Cancer Metastasis Rev 34: 511-525, 2015.

24. Held G, Murawski N, Ziepert M, Fleckenstein J, Pöschel V, Zwick C, Bittenbring J, Hänel M, Wilhelm S, Schubert J, et al: Role of radiotherapy to bulky disease in elderly patients with aggressive B-cell lymphoma. J Clin Oncol 32: 1112-1118, 2014.

This work is licensed under a Creative Commons Attribution-NonCommercial-NoDerivatives 4.0 International (CC BY-NC-ND 4.0) License. 